\title{
UNA NUEVA ESPECIE DE SWARTZIA (LEGUMINOSAE) DE COSTA RICA
}

\author{
Nelson A. Zamora ${ }^{1,2} \&$ Daniel Solano ${ }^{1}$ \\ ${ }^{1}$ Instituto Nacional de Biodiversidad (INBio), apdo. 22-3100, Santo Domingo, Heredia, Costa Rica \\ ${ }^{2}$ Organización para Estudios Tropicales (OET), apdo. 676-2050, San Pedro, Costa Rica
}

\begin{abstract}
A new species of Swartzia (Leguminosae), S. maquenqueana, from Northern Costa Rica is here described and illustrated.

Resumen. Se describe e ilustra la nueva especie Swartzia maquenqueana (Leguminosae), del norte de Costa Rica.

Palabras clave / Key words: Swartzia, Swartzia maquenqueana, Leguminosae, Costa Rica.
\end{abstract}

La flora del Refugio Nacional de Vida Silvestre Mixto Maquenque (Fig. 1), en la zona norte de Costa Rica, es de particular interés y posee una combinación de especies exclusiva de la región, favorecida por un clima muy húmedo con altas precipitaciones, pero con una estacionalidad determinada por uno o dos meses secos (Herrera \& Gómez 1993); además, en general la región cuenta con una topografía ondulada.

Las características de la vegetación han permitido identificarla como una región florística distinta respecto a regiones colindantes, como son las llanuras de Guatuso y de Tortuguero (ver Zamora et al. 2004); aglomera elementos de patrones florísticos vecinos, cuenta con un endemismo binacional (NicaraguaCosta Rica) en la parte baja media del Río San Juan, con especies como Eugenia sancarlosensis Barrie (Myrtaceae), Eschweilera costaricensis S.A. Mori (Lecythidaceae) y Stephanopodium costaricense Prance (Dichapetalaceae); también hay un endemismo local que se extiende hacia la parte sur del Corredor Biológico San Juan-La Selva, debido a recientes hallazgos de especies nuevas, como Faramea zamorensis Al. Rodr. (Rubiaceae), Drymonia glandulosa Kriebel, D. rubripilosa Kriebel (Gesneriaceae) y Symplocos striata Kriebel \& N. Zamora (Symplocaceae) (ver Rodríguez 2002, Kriebel \& Zamora 2004, Kriebel 2005, 2006).

El inventario de la flora del Refugio Maquenque inició, de forma más permanente e intensa, a partir de 2003 con las actividades del proyecto "Construyendo capacidad para Conservación de la Biodiversidad en Nicaragua y Costa Rica", financiado por la Iniciativa Darwin. El estudio florístico consistió principalmente en el establecimiento de parcelas de una hectárea en distintos bosques y otras exploraciones botánicas paralelas, aplicando el método de inventario libre o al azar, mediante recolectas generales de material reproductivo. Durante la ejecución del proyecto se recolectó material fértil y datos sobre abundancia de la especie que a continuación describimos.

\section{Swartzia maquenqueana N. Zamora \& D. Solano, sp. nova}

Arbor 12-30 $m$ alta; folia (petiolus et rachis comprehensi) 32-42.7 cm longa; foliola 9-13, oblanceolata vel obovata, discolora, subtus dense sericea; racemi cauliflori, 10-14.9 cm longi, bracteae 0.5-0.7 mm longae; pedicelli 4-8 mm; flos apetalus; legumen 5-14.2 cm longum, 2.1-2.5 cm latum, aurantiacum, pilosum, semina 1-4, elliptica.

Tipo: Costa Rica. Alajuela; San Carlos, Refugio Nacional de Vida Silvestre Mixto Maquenque, $6 \mathrm{~km}$ NE de Boca Tapada, cerca a Laguna del Lagarto Lodge, $500 \mathrm{~m}$ camino a Golfito, $10^{\circ} 41^{\prime} 10^{\prime \prime} \mathrm{N}, 84^{\circ} 10^{\prime} 50^{\prime \prime} \mathrm{W}, 90$ $\mathrm{m}, 11$ dic 2005 (fl), D. Solano, I. Mena \& D. Santamaría 2933 (Holotipo: INB, isotipos: CR, HULE, K, MO, PMA, USJ). Figs. 2, 3, 4.

Árbol, $12-30 \mathrm{~m}$ de alto y $25-45 \mathrm{~cm}$ de diámetro, el tronco acanalado con la corteza externa ligeramente escamosa y la interna delgada con secreción roja. Ramitas jóvenes con pubescencia densa, tomentosa ferrugínea; estípulas de 12 x $4 \mathrm{~mm}$, deltoides y decíduas. Hojas imparipinnadas, alternas, $33-42.7 \mathrm{~cm}$ de largo, folíolos 9-13, oblanceolados u obovados, opuestos, glabros en el haz, con pubescencia serícea densa (excepto en la vena central, tomentosa e inmersa) y cuando frescos con una coloración grisácea o glauca en el envés (pardo-pálida cuando secos), peciólulos de 3-5 mm de largo, los basales de 6.8-7.1 x 3.8-4.8 $\mathrm{cm}$, los medios de 11.2-16.6 x 5.1-7.9 cm y los distales de $14-15.9(-18.3) \times 6.3-8.5 \mathrm{~cm}$, ápice abrupto, cortoacuminado o agudo, redondeado o a veces truncado o levemente emarginado en los folíolos basales, base ligeramente asimétrica, venas secundarias 6-9 pares 
por lado; raquis de (15-)17.2-22.1 cm de largo, con una pubescencia tomentosa densa, levemente acanalado arriba; pecíolo de (3.4-) 4.1-4.5 (-6.7) cm de largo, cilíndrico o ligeramente surcado. Inflorescencias racemosas, péndulas, 10-14.9 cm de largo, saliendo en grupos de 3-6 racimos de las ramitas gruesas y de la parte defoliada de éstas; pedúnculo de (1.1-) 1.5-2.5 $(-3.5) \mathrm{cm}$ de largo, raquis y flores con una pubescencia serícea densa, dorada o pardo-dorada; brácteas de 0.5-0.7 mm de largo, decíduas, bractéolas ausentes. Botones florales de 3.5-6.5 x 2.5-5.1 mm; pedicelos de 4-8 $\mathrm{mm}$ de largo. Flores con cáliz de 8 a $9 \mathrm{~mm}$ de largo, con 4 ó 5 lóbulos, de forma irregular, pardo-seríceos externamente, glabros internamente, libres o cortamente fusionados, verde-crema, reflexos y persistentes; corola ausente; estambres numerosos, entre 30 y 35 , glabros, libres o levemente fusionados en la base, desiguales, los más largos con filamentos de 8 a $11 \mathrm{~mm}$ de largo, los más cortos con filamentos de 4 a $5 \mathrm{~mm}$ de largo; anteras de 0.7-1.1 x 0.5-0.7 mm, oblongas a elípticas, basifijas; gineceo con pubescencia pardo-velutina, ovario estipitado, cerca de $5 \mathrm{~mm}$ de largo (incluyendo el ginóforo), con hasta 13 óvulos, estilo $c a .3 \mathrm{~mm}$ de largo, arqueado, glabro, estigma truncado. Legumbres de 5-14.2 x 2.1-2.5 cm, moniliformes o constrictas entre las semillas, anaranjadas o amarillentas cuando maduras, cubiertas con una pubescencia sedosa densa, con el ápice terminando en una punta muy aguda y rígida, cuando secas con prominentes venas transversales irregulares, 1-4 semillas de 2.0-2.5 x $1.8-2.0 \mathrm{~cm}$, elipsoidales, negras, lustrosas y moteadas, dispuestas en forma oblicua y cubiertas con un arilo rojo intenso de textura pastosa.

DisTRIBUCIÓN, HÁBITAT Y ESTATUS DE CONSERVACIÓN. Swartzia maquenqueana se encuentra hasta ahora restringida al área del Refugio Maquenque (Fig.1), donde crece en bosques muy húmedos con terrenos mayormente ondulados o irregulares, entre 50 y $100 \mathrm{~m}$ de elevación. Se espera que se encuentre en Nicaragua, dada la cercanía de ese país. El descubrimiento de esta especie es de gran relevancia para apoyar las acciones actuales de conservación y manejo que se desarrollan en la región. Además, refleja que ésta cuenta con una biodiversidad importante aún desconocida.

Etimología. El epíteto maquenqueana se refiere al Refugio de Vida Silvestre Maquenque, única área donde se ha hallado la nueva especie.

Swartzia maquenqueana pertenece a la sección Swartzia, subsección Swartzia, serie Tounateae, que se caracteriza principalmente por la ausencia de pétalos y bractéolas (Cowan 1981). Esta nueva especie se distingue notablemente por el tronco acanalado, las hojas con raquis sin alas, acanalado en la parte superior y tomentoso, los folíolos oblanceolados u obovados, los basales claramente más pequeños que los distales, cóncavos cuando frescos, los basales más o menos erectos y los distales horizontales, bicoloros con el haz verde oscuro y el envés grisáceo o más o menos glauco y con una pubescencia tomentosa densa; los frutos constrictos entre las semillas, anaranjados o amarillentos cuando maduros, con la superficie tomentosa, y semillas con un arilo rojo intenso. En Costa Rica, S. maquenquena es la segunda especie descrita de esta serie taxonómica (sin pétalos ni bractéolas); la mayoría son especies sudamericanas.

Paratipos: Costa Rica. Alajuela: San Carlos, Cuenca del Río San Carlos, Pital, Yucatán, Finca Octubre 78, $10^{\circ} 38^{\prime} 50^{\prime \prime} \mathrm{N}, 84^{\circ} 12^{\prime} 15^{\prime \prime} \mathrm{W}, 100 \mathrm{~m}, 25$ ago 1995 (est), N. Zamora 2320 (INB); San Carlos, Cuenca del Río San Carlos, Finca Aserradero San Jorge, 10²' $57^{\prime \prime N}$, 84¹0'27'W, 100 m, 6 nov 1996 (est), N. Zamora, B. Hammel \& A. Rodríguez 2542 (INB); Refugio Nacional de Vida Silvestre Mixto Maquenque, $6 \mathrm{~km}$ NE de Boca Tapada, cerca a Laguna del Lagarto Lodge, $500 \mathrm{~m}$ camino a Golfito, $10^{\circ} 41^{\prime} 10^{\prime \prime} \mathrm{N}, 84^{\circ} 10^{\prime} 50^{\prime} \mathrm{W}$, 90 m, 10 ene 1997 (fr), N. Zamora, A. Zeledón y M. Ocampo 2559 (INB); 9 nov 2004 (fr), D. Solano \& B. Hernández 1438 (CR, INB, MO); 2 jun 2005 (fr), D. Solano, I. Mena \& B. Hernández 2498 (BM, CR, F, HULE, INB, K, MO, NY, PMA, USJ); 2 dic 2005 (fl), N. Zamora, D. Solano y I. Mena 3875 (INB).

Historia Natural. Las flores han sido observadas en diciembre y los frutos en enero, mayo, junio y noviembre. Tanto la floración como la fructificación son abundantes. Se ha observado el tucán Ramphastos swainsonii (Ramphastidae) comiendo el arilo de las semillas. Las ramitas son huecas y albergan hormigas del género Azteca (Formicidae) (Longino, com. pers.) En el Refugio Maquenque, un estudio realizado en 12 parcelas permanentes de una hectárea reveló que la especie tiene una densidad de un árbol por hectárea (Zamora et al., datos sin publicar). El género Swartzia se ha regsitrado como hospedero de la mariposa diurna Morpho helenor marinita (Nymphalidae, Morphinae, DeVries 1987). Además, algunas especies, como $S$. cubensis (Britton \& P. Wilson) Standl. y S. simplex (Sw.) Spreng., son plantas hospederas de algunas familias de lepidópteros, e.g. Apatelodidae, Arctiidae, Crambidae, Elachistidae, Gelechiidae, Geometridae, 
Hesperiidae, Limacodidae, Noctuidae, Notodontidae, Nymphalidae, Pieridae, Pyralidae, Riodinidae, Saturniidae, Thyrididae y Tortricidae; también son alimento de adultos de escarabajos (e.g. Chrysomelidae y Scarabaeidae) y de chinches de la familia Coreidae (Janzen \& Hallwachs 2006).

Agradecimientos. Expresamos nuestro agradecimiento a la compañera Claudia Aragón, por su excelente ilustración, a la Iniciativa Darwin, por el financiamiento a través del proyecto "Construyendo capacidad para Conservación de la Biodiversidad en Nicaragua y Costa Rica" (No. 162/12/020), que permitió el trabajo de campo y de laboratorio. Además, al proyecto Flora Digital de La Selva (OET/MOBOT/CR-USA/NSF) que apoyó, en parte, el trabajo de campo.

\section{LiTERATURA CITADA}

Cowan, R.S. 1968. Swartzia (Leguminosae: Caesalpinioideae, Swartzieae). Flora Neotropica, Monogr. 1: 1-228.

DeVries, P.J. 1987. The butterflies of Costa Rica and their natural history. Papilionidae, Pieridae, Nymphalidae.
Princeton Univ. Press, New Jersey. 327 p.

Herrera, W. \& L.D. Gómez. 1993. Mapa de unidades bióticas de Costa Rica. U.S. Fish \& Wildlife Serv. / The Nature Conservancy.

Kriebel, R. \& N. Zamora. 2004. Symplocos striata (Symplocaceae), una nueva especie de la vertiente caribe de Costa Rica. Lankesteriana 4(3): 17-174.

Kriebel, R. 2005. Una nueva especie y un nuevo registro de Drymonia (Gesneriaceae) en Costa Rica. Lankesteriana 5(1): 81-83.

Kriebel, R. 2006. A new species and notes on Drymonia (Gesneriaceae) from Costa Rica. Novon 16: 65-68.

Rodríguez, A. 2002. A new species of Faramea (Rubiaceae) from Costa Rica. Novon 12(4): 536-538.

Zamora, N., B. Hammel \& M.H. Grayum. 2004. Vegetación. In: Hammel, B.E., M.H. Grayum, C. Herrera \& N. Zamora (eds.). Manual de Plantas de Costa Rica. Vol. I. Introducción. Monogr. Syst. Bot. Missouri Bot. 97: 1-300.

\section{Sitio de Internet Consultado:}

Janzen, D.H. \& W. Hallwachs. 2005. Caterpillars, pupae, butterflies \& moths of the Area de Conservación Guanacaste, northwestern Costa Rica. http://Janzen. sas.upenn.edu/caterpillars/database.lasso

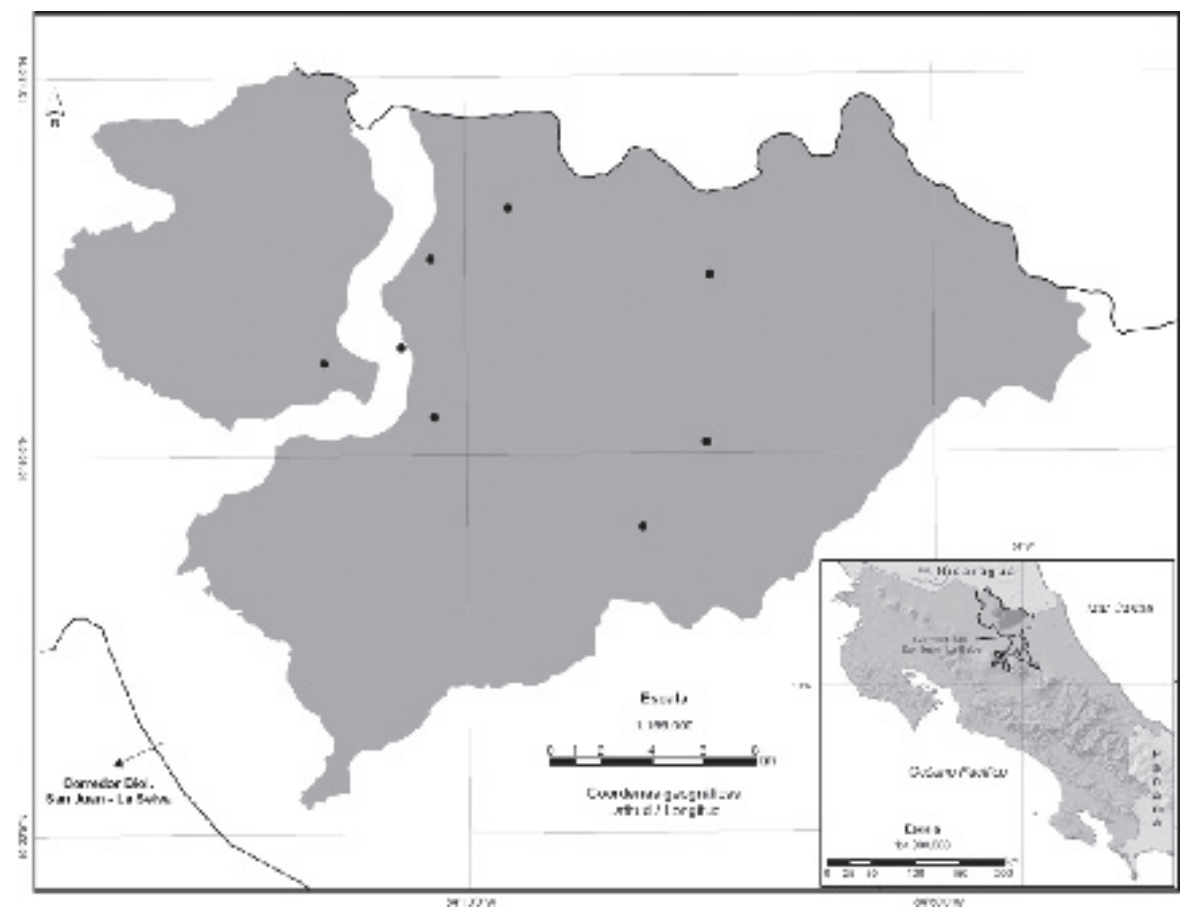

Fig. 1. Ubicación del Refugio de Vida Silvestre Maquenque, en la zona norte de Costa Rica. Los puntos indican la distribución conocida de Swartzia maquenqueana. Mapa elaborado por Marcia Snyder (OET). 


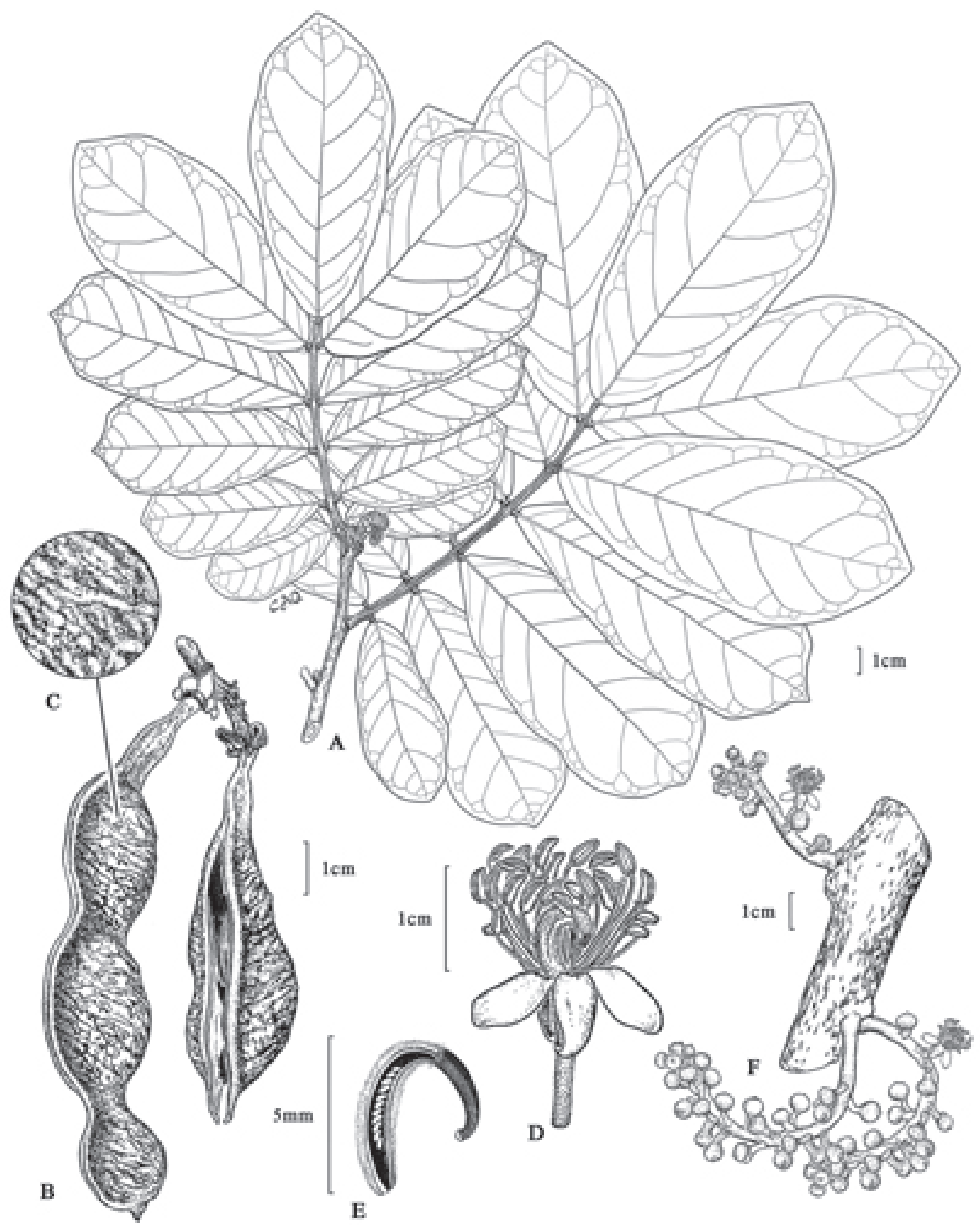

Fig. 2. Swartzia maquenqueana N. Zamora \& D. Solano. A. Rama. B. Frutos. C. Detalle de la superficie del fruto. D. Flor. E. Ovario. F. Inflorescencias. (A, D-F del tipo: Solano et al. 2933; B de Zamora et al. 2559). Ilustraciones de Claudia Aragón. 


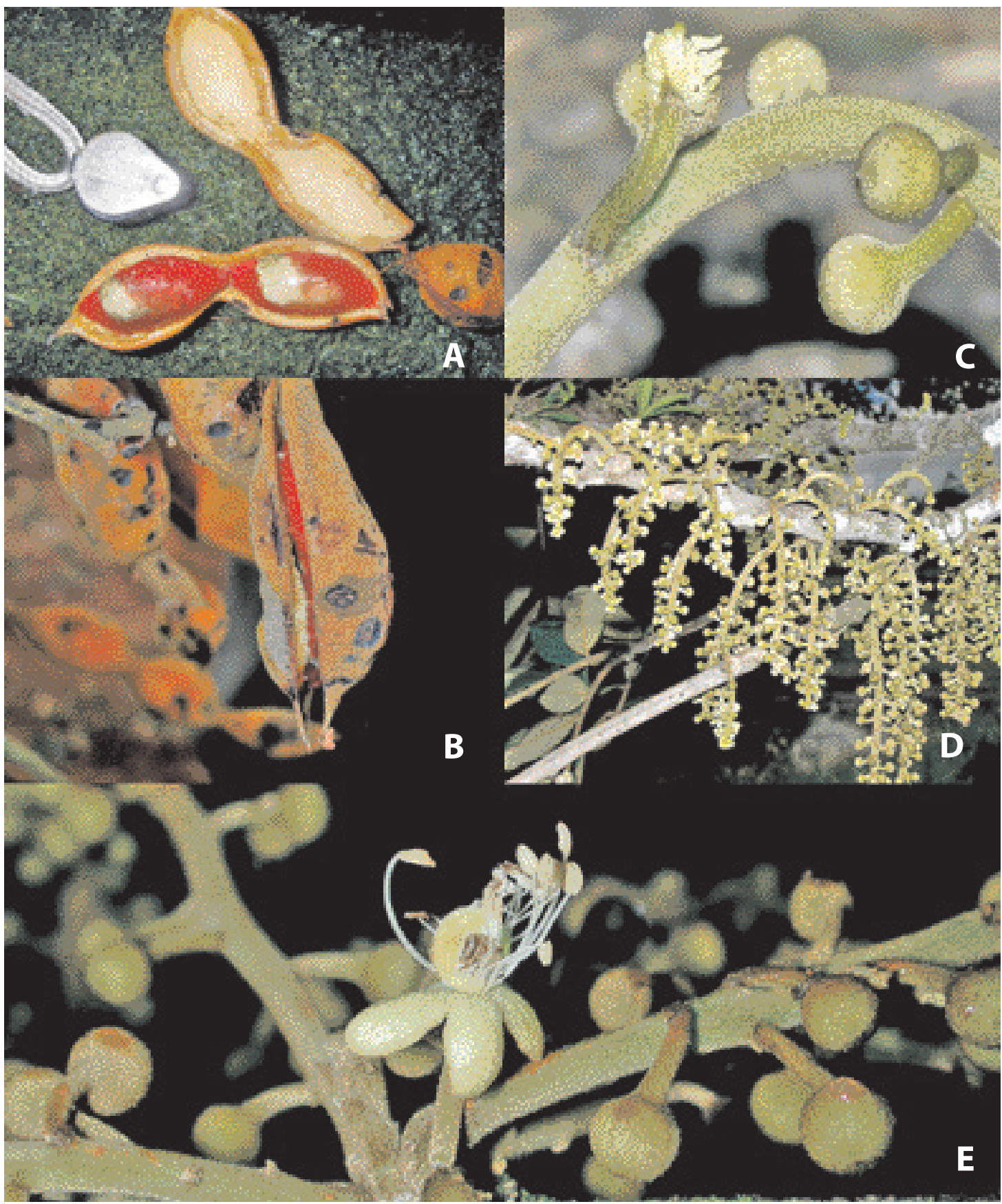

Fig. 3. Swartzia maquenqueana N. Zamora \& D. Solano. A. Fruto abierto, con semillas cubiertas por arilo rojo. B. Rama con frutos maduros. C. Detalle de inflorescencia. D. Rama con inflorescencias. E. Flor en antesis. Fotos de los autores. 


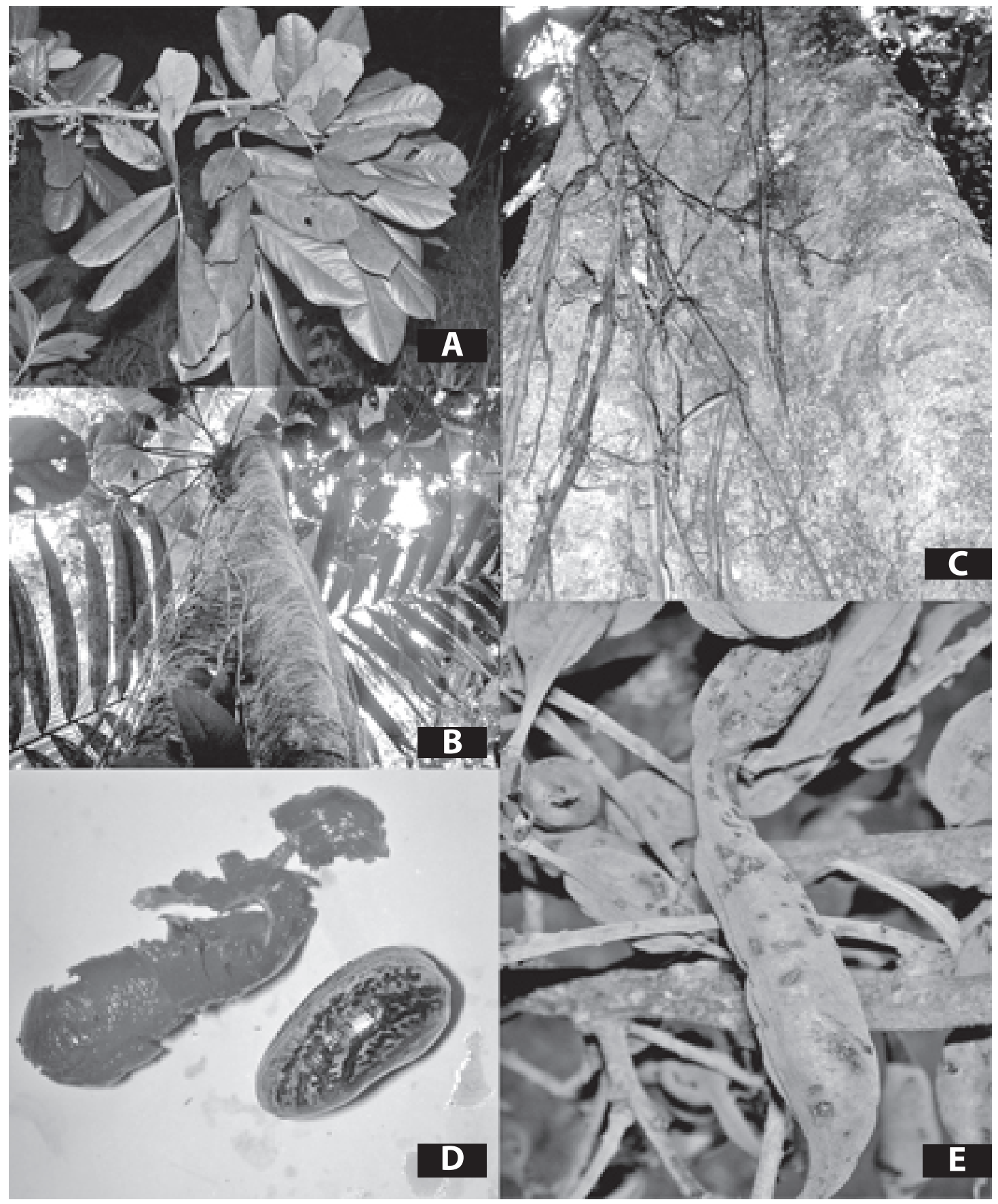

Fig. 4. Swartzia maquenqueana N. Zamora \& D. Solano. A. Aspecto del follaje. B. Aspecto del tronco. C. Detalle del tronco. D. Arilo y semilla. E. Rama con frutos. Fotos de los autores. 